\title{
Clinical utility of prostate-specific antigen mass ratio for prediction of prostate cancer detection on a repeated prostate biopsy
}

\author{
Won Ki Lee', Sangchul Lee², Sung Kyu Hong², Sang Eun Lee², Won Suk Choi ${ }^{3}$, Seok-Soo Byun² \\ ${ }^{1}$ Department of Urology, Hallym University Chuncheon Sacred Heart Hospital, Chuncheon; ${ }^{2}$ Department \\ of Urology, Seoul National University Bundang Hospital, Seongnam and ${ }^{3}$ Department of Urology, Choi \\ Won Suk Urology Clinic, Yongin, Korea
}

\section{ABSTRACT}

Purpose: To assess the clinical utility of the prostate-specific antigen mass ratio (PSA-MR), a newly developed PSA derivative, simply defined as the (i) PSA density (PSA-D) multiplied by the plasma volume or (ii) total PSA amount in circulation per prostate volume, for predicting prostate cancer ( $\mathrm{PCa}$ ) among men undergoing repeated prostate biopsy (PBx).

Materials and Methods: Patients $(\mathrm{n}=286)$, who underwent a repeated $\mathrm{PBx}$, were analyzed. The various parameters associated with PCa detection were noted in each patient. PSA-MR was also calculated.

Results: PCa was detected in $63(22.0 \%)$ of 286 patients. PSA-MR was the independent predictor in the univariate- and multivariate logistic regression analyses $(\mathrm{OR}=3.448, \mathrm{p}$ $=0.001$ and $\mathrm{OR}=13.430, \mathrm{p}=0.033$, respectively). A nomogram that incorporated PSA-MR was considered a useful tool (predictive accuracy: 79.2\%, 95\% CI: 0.726-0.858, $\mathrm{p}<0.001$ ). Furthermore, a nomogram that incorporated PSA-MR would have avoided $59.6 \%$ of unnecessary repeated PBx. The predictive accuracy of PSA-MR was also superior to that of PSA or PSA-D ( $\mathrm{p}=0.013$ and 0.009 , respectively).

Conclusions: PSA-MR was an independent predictor, and its consideration would have avoided 59.6\% of unnecessary repeated PBx for PCa detection. PSA-MR was also superior than PSA or PSA-D. Our results support the use of PSA-MR to facilitate counseling with patients after a negative initial PBx, and use of PSA-MR might reduce further unnecessary biopsies.

\section{ARTICLE INFO}

\section{Key words:}

Prostatic Neoplasms; Prostate-

-Specific Antigen; Biopsy; Mass

Screening

Int Braz J Urol. 2014; 40: 484-92

Submitted for publication:

August 18, 2013

Accepted after revision:

October 09, 2013

\section{INTRODUCTION}

Thirty percent of prostate cancer $(\mathrm{PCa})$ cases are unfortunately missed during the initial prostate biopsy $(\mathrm{PBx})$, although $\mathrm{PBx}$ is the diagnostic procedure of choice for $\mathrm{PCa}$ detection $(1,2)$. Since negative initial $\mathrm{PBx}$ cannot conclusively exclude the presence of $\mathrm{PCa}$, many men undergo repeated
PBx. However, not all men have a high risk of $\mathrm{PCa}$ after a negative initial PBx (3), and repeated PBx could increase the risks of $\mathrm{PBx}$-associated complications such as infection. Furthermore, repeated PBX could be associated with high economic and psychological costs. Therefore, it is important to predict $\mathrm{PCa}$ after a negative initial $\mathrm{PBx}$ in order to reduce further unnecessary biopsies. 
Various clinical and pathological findings have historically been considered risk factors for PCa after a negative initial PBx. These include age, digital rectal examination (DRE), prostate volume, prostate-specific antigen (PSA), and its derivatives such as PSA velocity (PSA-V) and PSA density (PSA-D), high grade prostatic intraepithelial neoplasia (HGPIN), and atypical small acinar proliferation (ASAP) (4). Among these risk factors, PSA-related parameters are widely used to predict PCa after a negative initial PBx. However, clinical evidence is lacking with regard to the ability of PSA-related parameters to identify patients for whom repeated PBx is warranted $(5,6)$.

The parameter called the PSA mass ratio (PSA-MR) was recently suggested as a PCa screening tool by Choi et al. (7). One of the biggest drawbacks of the PSA test is that various factors can influence the PSA level (8-12). Therefore, the authors examined the effects of various factors on PSA levels in a screening population, and finally devised the PSA-MR. PSA-MR is simply defined as the (i) PSA-D multiplied by the plasma volume or (ii) total PSA amount in circulation per prostate volume. The authors suggested that the PSA-MR might be more stable than PSA, when the body mass index (BMI), plasma volume, and prostate volume change.

The present study assessed the clinical utility of PSA-MR for predicting PCa among men undergoing repeated $\mathrm{PBX}$.

\section{MATERIALS AND METHODS}

After receiving approval from the relevant institutional review board, a PBx database maintained at a single institution was reviewed. Between May 2003 and March 2011, a total of 292 patients underwent a repeated multi $(\geq 12)$-core transrectal ultrasonography (TRUS)-guided PBx after a negative initial PBx. A repeated PBx was performed based on suspicious findings on DRE $(\mathrm{n}=17)$, TRUS $(\mathrm{n}=46)$, previous ASAP $(\mathrm{n}=35)$, HGPIN $(\mathrm{n}=15)$ and/or PSA $>4.0 \mathrm{ng} / \mathrm{mL}(\mathrm{n}=263)$. Among these patients, those who had undergone prior PBx at other institutions, who had been surgically treated for prostatic disease prior to repeated $\mathrm{PBx}$, or who were taking medication known to influence PSA levels such as $5-\alpha$ reductase inhi- bitors were excluded. Finally, 286 patients were included in this study.

The parameters noted for each patient were age, BMI, DRE and TRUS findings, prostate volume, number of cores removed, interval between initial and repeated $\mathrm{PBx}$, pathologic characteristics of initial PBx such as HGPIN and ASAP, and PSA, and its derivatives such as the percentage of free PSA (\%fPSA), PSA-V, PSA doubling time (PSA-DT), and PSA-D. PSA-MR was also calculated and noted for each patient.

DRE and TRUS findings were classified as normal or suspicious for PCa. The prostate volume was routinely measured according to the prolate ellipsoid formula via TRUS before undergoing PBX. The prostate was routinely bilaterally biopsied near the apex, mid-gland, and base, with 6 biopsies per side. Suspicious lesions were additionally biopsied. Pathologic PBx findings were assessed by the referred pathologist. The \%fPSA was calculated as the ratio of free-to-total PSA, multiplied by 100 . PSA-V was calculated based on the slope obtained by fitting a linear regression analysis, using all PSA values and the equation: $p=a t+b$ (13). PSA-DT was calculated based on the natural logarithm of 2-divided by the slope obtained through fitting a linear regression of the natural logarithm of PSA on time (14). PSA-D was calculated as the PSA value divided by the prostate volume.

PSA-MR $(\mu \mathrm{g} / \mathrm{mL})$ was defined as the (i) PSA-D multiplied by the plasma volume or (ii) the total PSA amount in circulation per prostate volume. Body surface area, plasma volume, PSA mass, and PSA-MR were estimated using the following equations (7):

Body surface area $\left(\mathrm{m}^{2}\right)=$ body weight $(\mathrm{kg})^{0.425} \mathrm{x}$ height $(\mathrm{m})^{0.725} \mathrm{x} 0.007184$ (15).

Plasma volume $(\mathrm{l})=$ body surface area $\left(\mathrm{m}^{2}\right)$ x 1.670 (16).

PSA mass $(\mu \mathrm{g})=$ PSA $(\mathrm{ng} / \mathrm{mL}) \times$ plasma volume (l) (17).

PSA-MR $(\mu \mathrm{g} / \mathrm{mL})=$ PSA mass $(\mu \mathrm{g}) /$ prostate volume (mL).

To compare the parameter distributions according to the presence of $\mathrm{PCa}$ in a repeated $\mathrm{PBx}$ cohort, Student's t-test or Mann-Whitney test and 
Chi-square test were used in the comparison of continuous and categorical variables, respectively. A univariate logistic regression analysis was used to assess the associations between the parameters and PCa prediction on repeated PBx. Subsequently, a multivariate logistic regression analysis was performed with the statistically significant parameters from the univariate analysis, in order to assess the independence of PSA-MR. PSA-D was excluded in the multivariate analysis that incorporated PSA-MR, due to multi-collinearity issue (tolerance value $=0.303$ ). Nomograms that did and did not incorporate PSA-MR were generated with the multivariate regression coefficients. Next, receiver operating characteristic (ROC)-derived areas under the curves (AUCs) were estimated and compared with the DeLong et al. test. The ROC curve cut-off value was estimated with the maximum Youden index. Furthermore, the sensitivity, specificity, positive predictive value (PPV) and negative predictive value (NPV) were calculated, and compared with the proportional paired test. The proportion of avoided unnecessary repeated $\mathrm{PBx}(\mathrm{PAB})$ was also estimated with the following equation (5): (number of false positives nomogram not incorporating PSA-MR - number of false positives nomogram incorporating PSA-MR) $\div$ number of false positives nomogram not incorporating PSA-MR. At fixed sensitivity thresholds, which were set at 90 and 95\%, the specificity, PPV, NPV and PAB were also calculated and compared, in order to specifically simulate the requirements of a PSA-MR.

To compare PSA-MR individually with PSA and other derivatives, ROC-AUCs were used and compared with the DeLong et al. test.

All $p$ values were 2 -sided, and $p<0.05$ was considered significant.

\section{RESULTS}

Table-1 shows the descriptive characteristics of the 286 patients who underwent a repeated PBx. PCa was detected in 63 (22.0\%) of these 286 patients. Patients with $\mathrm{PCa}$ differed significantly from those without $\mathrm{PCa}$ in several parameters. $\mathrm{Pa}-$ tients with PCa tended to be older $(p=0.004)$ and to have higher suspicious DRE finding ratios ( $p=$ $0.026)$, smaller prostates $(p=0.001)$, and greater previous ASAP ratios $(p=0.001)$. The mean PSA-
-V and PSA-MR levels were also higher in patients with PCa ( $p=0.001$ and 0.025 , respectively). In contrast, there was no association with respect to PSA ( $p=0.526)$, \%fPSA ( $p=0.084)$, PSA-DT $(p=$ $0.421)$, or PSA-D ( $p=0.306)$.

In univariate logistic regression analyses (Table-2), age (odds ratio $(\mathrm{OR})=1.060, \mathrm{p}=0.005$ ), a suspicious DRE finding (OR $=3.359, \mathrm{p}=0.025)$, prostate volume $(\mathrm{OR}=0.973, \mathrm{p}=0.003)$, previous ASAP (OR $=5.547, p<0.001)$, PSA (OR $=1.038, p$ $=0.038)$, PSA-V $(O R=1.066, p=0.019)$, and PSA-D (OR $=7.399, p=0.049)$ were significantly associated with the repeated PBX outcome. PSA-MR was also significantly associated with outcome (OR $=3.448, p=0.001)$. A multivariate logistic regression model that did not incorporate PSA-MR showed that age $(\mathrm{OR}=1.090, \mathrm{p}=0.001)$, prostate volume (OR $=0.940, \mathrm{p}<0.001)$, and previous ASAP $(\mathrm{OR}=7.261, \mathrm{p}<0.001)$ were independent predictors of the repeated PBx outcome (Table-3). When PSA-MR was incorporated into the multivariate logistic regression model, age and previous ASAP remained independent predictors $(\mathrm{OR}=1.115$ and 8.946, respectively; $\mathrm{p}<0.001$ for both). PSA-MR was also an independent predictor of the repeated $\mathrm{PBx}$ outcome (OR $=13.430, \mathrm{p}=0.033$; Table-3).

A multivariate model that incorporated PSA-MR was considered a useful tool (predictive accuracy $=79.2 \%, 95 \% \mathrm{CI}=0.726-0.858, \mathrm{p}<$ 0.001). The model that incorporated PSA-MR was not inferior with respect to predictive accuracy ( $\mathrm{p}$ $=0.853)$, sensitivity $(p=0.143)$, PPV $(p=0.063)$ or NPV ( $p=0.901)$, when compared with the model that did not incorporate PSA-MR. Furthermore, the model that incorporated PSA-MR was superior with respect to specificity ( $p<0.001)$, and use of this model would have avoided 59.6\% of unnecessary repeated PBx (Table-4). At fixed sensitivity thresholds of 90\% and 95\%, use of the model that incorporated PSA-MR would also have avoided 9.2\% and 5.9\% of unnecessary repeated PBx, respectively (Table-4).

When compared individually with the PSA-related parameters, the predictive accuracy of PSA-MR was 59.2\% (95\% CI $=0.506-0.678$, $\mathrm{p}=0.025$ ), which was superior to those of PSA, \%fPSA, PSA-DT or PSA-D ( $\mathrm{p}=0.013,0.022,0.019$, and 0.009, respectively; Table-5). 
Table 1 - Associations of different variables according to the presence of prostate cancer in the entire repeated biopsy cohort.

\begin{tabular}{|c|c|c|c|c|}
\hline Variables & $\begin{array}{l}\text { Total cohort } \\
(\mathrm{n}=286)\end{array}$ & $\begin{array}{l}\text { Prostate cancer } \\
\qquad(n=63)\end{array}$ & $\begin{array}{l}\text { No prostate Cancer } \\
\qquad(\mathrm{n}=223)\end{array}$ & $p$ value \\
\hline Age, years, mean \pm SD & $64.5 \pm 7.8$ & $67.0 \pm 6.7$ & $63.9 \pm 7.9$ & 0.004 \\
\hline $\mathrm{BMI}, \mathrm{kg} / \mathrm{m}^{2}$, mean $\pm \mathrm{SD}$ & $24.3 \pm 2.3$ & $24.2 \pm 2.3$ & $24.4 \pm 2.3$ & 0.600 \\
\hline Suspicious DRE, n (\%) & $15(5.2)$ & $7(11.1)$ & $8(3.6)$ & 0.026 \\
\hline Suspicious TRUS, n (\%) & $45(15.7)$ & $12(20.7)$ & $33(15.4)$ & 0.338 \\
\hline Prostate volume, $\mathrm{mL}$, mean $\pm \mathrm{SD}$ & $50.5 \pm 21.9$ & $43.4 \pm 16.2$ & $52.6 \pm 22.9$ & $0.001^{*}$ \\
\hline No. biopsy cores, mean \pm SD & $13.2 \pm 1.2$ & $13.1 \pm 1.1$ & $13.3 \pm 1.2$ & 0.317 \\
\hline $\begin{array}{l}\text { II Interval from previous biopsy, months, } \\
\text { mean } \pm \text { SD }\end{array}$ & $27.2 \pm 19.0$ & $26.2 \pm 22.3$ & $27.4 \pm 17.9$ & $0.214^{*}$ \\
\hline Previous ASAP, n (\%) & $33(11.5)$ & $18(28.6)$ & $15(6.7)$ & 0.001 \\
\hline Previous HGPIN, n (\%) & $10(3.5)$ & $3(4.8)$ & $7(3.1)$ & 0.463 \\
\hline $\mathrm{PSA}, \mathrm{ng} / \mathrm{mL}$, mean $\pm \mathrm{SD}$ & $9.99 \pm 7.21$ & $11.73 \pm 10.94$ & $9.50 \pm 5.67$ & $0.526^{*}$ \\
\hline$\% \mathrm{fPSA}, \%$, mean \pm SD & $19.65 \pm 10.64$ & $15.52 \pm 6.94$ & $20.99 \pm 11.33$ & 0.084 \\
\hline $\mathrm{PSA}-\mathrm{V}, \mathrm{ng} / \mathrm{mL} / \mathrm{year}$, mean $\pm \mathrm{SD}$ & $2.58 \pm 4.63$ & $3.87 \pm 6.09$ & $2.21 \pm 4.07$ & $0.001^{*}$ \\
\hline PSA-DT, months, mean \pm SD & $83.40 \pm 176.46$ & $83.48 \pm 173.47$ & $83.38 \pm 177.84$ & $0.421^{*}$ \\
\hline $\mathrm{PSA}-\mathrm{D}, \mathrm{ng} / \mathrm{mL} / \mathrm{mL}$, mean $\pm \mathrm{SD}$ & $0.23 \pm 0.13$ & $0.26 \pm 0.17$ & $0.22 \pm 0.12$ & $0.306^{*}$ \\
\hline $\mathrm{PSA}-\mathrm{MR}, \mathrm{ug} / \mathrm{mL}$, mean $\pm \mathrm{SD}$ & $0.49 \pm 0.38$ & $0.65 \pm 0.59$ & $0.45 \pm 0.28$ & $0.025^{*}$ \\
\hline
\end{tabular}

$\mathbf{S D}=$ standard deviation; $\mathbf{I Q R}$ = interquartile range; $\mathbf{B M I}=$ body mass index, $\mathbf{D R E}=$ digital rectal examination; $\mathbf{T R U S}=$ transrectal ultrasonography; No. = number, $\mathbf{A S A P}$ = atypical small acinar proliferation; HGPIN = high grade prostatic intraepithelial neoplasia; PSA = prostate-specific antigen; \% fPSA = percent of free prostate-specific antigen; $\mathbf{P S A}-\mathbf{V}=$ prostate-specific antigen velocity; $\mathbf{P S A}-\mathbf{D T}=$ prostate-specific antigen doubling time; $\mathbf{P S A}-\mathbf{D}=$ prostate-specific antigen density, $\mathbf{P S A}-\mathbf{M R}=$ prostatespecific antigen mass ratio.

${ }^{*}$ Non-parametric comparison.

\section{DISCUSSION}

After the introduction of the PSA test in the 1980s (18), PSA is among the most widely used parameters for predicting PCa detection. Various PSA derivatives were also devised to enhance the predictive accuracy for PCa detection, and these have also become widely used (4). However, clinical evidence regarding the efficacy of PSA and its derivatives to identify $\mathrm{PCa}$ in patients referred for a repeated PBx is still lacking $(5,6)$, although the clinical utilities of these parameters in guiding repeated PBx decisions have been reported $(2,19-21)$.
The biggest drawback of the PSA test is its low predictive accuracy for PCa detection. Since PSA is not a PCa-specific marker, elevated blood levels could be driven by various conditions (8). PSA is influenced by the prostate size or androgens $(8,9)$. Age, ethnicity, and obesity also influence PSA levels (1012). None of the currently used PSA cut-offs consistently identify PCa and exclude non-PCa (11). Concerns regarding the predictive accuracy of PSA have led investigators to evaluate additional methods of PSA analysis. However, as mentioned, these methods still have limitations with regard to clinical utility for the guidance of repeated PBx decisions $(5,6)$. 
Table 2 - Univariate logistic regression analyses of predictors of prostate cancer detection during a repeated biopsy.

\begin{tabular}{lccc}
\hline Variables & OR & $95 \% \mathrm{Cl}$ & $\mathrm{p}$ value \\
\hline Age & 1.060 & $1.018-1.104$ & 0.005 \\
BMI & 0.968 & $0.859-1.092$ & 0.599 \\
Suspicious DRE & 3.359 & $1.168-9.659$ & 0.025 \\
Suspicious TRUS & 1.431 & $0.686-2.986$ & 0.340 \\
Prostate volume & 0.973 & $0.956-0.991$ & 0.003 \\
No. biopsy cores & 0.881 & $0.689-1.128$ & 0.316 \\
Interval from previous biopsy & 0.996 & $0.981-1.012$ & 0.652 \\
Previous ASAP & 5.547 & $2.601-11.827$ & $<0.001$ \\
Previous HGPIN & 1.543 & $0.387-6.148$ & 0.539 \\
PSA & 1.038 & $1.002-1.075$ & 0.038 \\
\%fPSA & 0.929 & $0.854-1.011$ & 0.088 \\
PSA-V & 1.066 & $1.011-1.125$ & 0.019 \\
PSA-DT & 1.000 & $0.998-1.002$ & 0.997 \\
PSA-D & 7.399 & $1.008-54.286$ & 0.049 \\
PSA-MR & 3.448 & $1.670-7.115$ & 0.001 \\
\hline
\end{tabular}

$\mathbf{O R}=$ odds ratio; $\mathbf{C I}=$ confidence interval; $\mathbf{B M I}=$ body mass index; $\mathbf{D R E}=$ digital rectal examination; $\mathbf{T R U S}=$ transrectal ultrasonography; No. = number; $\mathbf{A S A P}=$ atypical small acinar proliferation; HGPIN = high grade prostatic intraepithelial neoplasia; $\mathbf{P S A}=$ prostate-specific antigen; $\% \mathbf{f P S A}=$ percent of free prostate-specific antigen; $\mathbf{P S A}-\mathbf{V}=$ prostate-specific antigen velocity; PSA-DT = prostate-specific antigen doubling time; PSA-D = prostate-specific antigen density; PSA-MR = prostate-specific antigen mass ratio.

Table 3 - Multivariate logistic regression analyses of predictors of prostate cancer detection during repeated biopsy, according to the incorporation of PSA mass ratio.

\begin{tabular}{lcccccc}
\hline \multirow{2}{*}{ Variables } & \multicolumn{3}{c}{ No incorporation of PSA-MR } & \multicolumn{3}{c}{ Incorporation of PSA-MR } \\
\cline { 2 - 7 } & OR & $95 \% \mathrm{Cl}$ & $\mathrm{p}$ value & OR & $95 \% \mathrm{Cl}$ & $\mathrm{p}$ value \\
\hline Age & 1.090 & $1.038-1.145$ & 0.001 & 1.115 & $1.058-1.174$ & $<0.001$ \\
Suspicious DRE & 2.927 & $0.877-9.769$ & 0.081 & 2.812 & $0.830-9.533$ & 0.097 \\
Prostate volume & 0.940 & $0.911-0.970$ & $<0.001$ & 0.976 & $0.945-1.008$ & 0.142 \\
Previous ASAP & 7.261 & $3.028-17.407$ & $<0.001$ & 8.946 & $3.683-21.732$ & $<0.001$ \\
PSA & 1.104 & $1.000-1.218$ & 0.050 & 0.936 & $0.826-1.062$ & 0.306 \\
PSA-V & 1.037 & $0.959-1.121$ & 0.368 & 1.026 & $0.943-1.116$ & 0.548 \\
PSA-D & 0.050 & $0.001-8.628$ & 0.255 & $*$ & & \\
PSA-MR & - & - & - & 13.430 & $1.240-145.478$ & 0.033 \\
\hline
\end{tabular}

$\mathbf{O R}=$ odds ratio; $\mathbf{C l}$ = confidence interval; $\mathbf{D R E}=$ digital rectal examination; $\mathbf{A S A P}=$ atypical small acinar proliferation; $\mathbf{P S A}=$ prostate-specific antigen; $\mathbf{P S A}-\mathbf{V}=$ prostatespecific antigen velocity; PSA-D = prostate-specific antigen density; PSA-MR = prostate-specific antigen mass ratio.

* PSA-D was excluded in the analysis that incorporated PSA-MR, due to a multicollinearity issue (tolerance value: 0.303 ). 
Table 4 - Comparison of the utilities of nomograms for the prediction of prostate cancer during repeated biopsy, according to the incorporation of the prostate-specific antigen mass ratio.

\begin{tabular}{|c|c|c|c|c|}
\hline Criteria & Variables & No inclusion of PSA-MR & Inclusion of PSA-MR & $p$ value \\
\hline \multirow[t]{6}{*}{ By Youden index* } & Threshold value & 0.227 & 0.367 & \\
\hline & Sensitivity (\%) & 68.3 & 54.0 & 0.143 \\
\hline & Specificity (\%) & 77.1 & 91.0 & $<0.001$ \\
\hline & PPV (\%) & 45.7 & 62.9 & 0.063 \\
\hline & NPV (\%) & 89.6 & 87.5 & 0.901 \\
\hline & PAB $(\%)$ & Reference & 59.6 & \\
\hline \multirow[t]{5}{*}{ At $90 \%$ sensitivity } & Threshold value & 0.100 & 0.103 & \\
\hline & Specificity (\%) & 36.8 & 42.6 & 0.245 \\
\hline & PPV (\%) & 28.8 & 30.8 & 0.752 \\
\hline & NPV (\%) & 93.2 & 94.1 & 0.880 \\
\hline & $\operatorname{PAB}(\%)$ & Reference & 9.2 & \\
\hline \multirow[t]{5}{*}{ At $95 \%$ sensitivity } & Threshold value & 0.087 & 0.087 & \\
\hline & Specificity (\%) & 31.8 & 36.3 & 0.369 \\
\hline & PPV $(\%)$ & 28.3 & 29.7 & 0.837 \\
\hline & NPV $(\%)$ & 95.9 & 96.4 & 0.993 \\
\hline & PAB (\%) & Reference & 5.9 & \\
\hline
\end{tabular}

$\mathbf{P S A}=$ prostate-specific antigen, $\mathbf{P S A}-\mathbf{M R}=$ prostate-specific antigen mass ratio, $\mathbf{P P V}=$ positive predictive value, $\mathbf{N P V}=$ negative predictive value, $\mathbf{P A B}=$ proportion that avoided unnecessary repeated prostate biopsy.

${ }^{*}$ Threshold value was estimated using the Youden index maximum.

Table 5 - Comparison of predictive accuracies of prostate cancer detection for the prostate-specific antigen and its derivatives with the receiver-operating characteristic-derived area under the curve.

\begin{tabular}{lcccc}
\hline Variables & ROC-derived AUC & $95 \% \mathrm{Cl}$ & $\begin{array}{c}\text { p value of } \\
\text { AUC }\end{array}$ & $\begin{array}{c}\text { p value of comparison with AUC } \\
\text { for PSA-MR }\end{array}$ \\
\hline PSA-MR & 0.592 & $0.506-0.678$ & 0.025 & Reference \\
PSA & 0.526 & $0.438-0.614$ & 0.526 & 0.013 \\
$\%$ fPSA & 0.361 & $0.204-0.518$ & 0.108 & 0.022 \\
PSA-V & 0.641 & $0.569-0.713$ & 0.001 & 0.013 \\
PSA-DT & 0.474 & $0.391-0.557$ & 0.527 & 0.019 \\
PSA-D & 0.542 & $0.456-0.628$ & 0.306 & 0.009 \\
\hline
\end{tabular}

AUC = area under the curve; $\mathbf{C I}=$ confidence interval; $\mathbf{R O C}=$ receiver operating characteristics; $\mathbf{P S A}-\mathbf{M R}=$ prostate-specific antigen mass ratio; $\mathbf{P S A}=$ prostate-specific antigen; \%fPSA = percent of free prostate-specific antigen; PSA-V = prostate-specific antigen velocity; $\mathbf{P S A}-\mathbf{D T}=$ prostate-specific antigen doubling time; $\mathbf{P S A}-\mathbf{D}=$ prostate-specific antigen density. 
The use of PSA-MR, the newly devised PSA derivative for PCa prediction, was recently proposed by Choi and colleagues (7). The authors examined the effects of plasma volume, insulin resistance (IR), prostate volume, and BMI on PSA in a general screening population after adjusting for potentially influencing factors, and finally devised the PSA-MR. This study is noteworthy because few studies have considered the combined effects of these variables on PSA levels. The authors suggested that PSA-MR might be more stable than PSA, when the BMI, plasma volume, and prostate volume change.

PSA-MR seems to be more attractive than other PSA-related parameters, because it considers confounding effects on PSA levels. Our study is the first to assess the clinical utility of PSA-MR for the prediction of $\mathrm{PCa}$ among men undergoing repeated $\mathrm{PBx}$, and we have identified the possible clinical utility of the PSA-MR. Currently PSA-MR is an independent predictor of PCa detection, even after adjusting for various parameters. The predictive accuracy of PSA-MR was also superior to that of PSA or most of its derivatives, including PSA-D. It is especially noteworthy that PSA-MR had a higher predictive accuracy than PSA-D, since PSA-MR is heavily correlated with PSA-D. This result suggests that PSA-MR might be a more valuable predictor than PSA-D, and might potentially replace PSA-D in the clinical setting. Furthermore, the model that incorporated PSA-MR was superior with respect to specificity, and its use would have avoided 59.6\% of unnecessary repeated PBx. Even when compared at fixed sensitivity thresholds of 90\% and 95\%, which were specifically applied to simulate the requirement for a PSA-MR to allow false-negative rates of only $10 \%$ or $5 \%$, use of the model that incorporated PSA-MR would have avoided 9.2\% and 5.9\% of unnecessary repeated PBx, respectively. Since none of predictors can be solely used to counsel an individual patient on the need to perform a $\mathrm{PBx}$ to rule out PCa (1), PSA-MR could potentially help physicians to counsel patients to pursue further PBx.

Recently, Hong et al. (22) investigated the value of PSA-MR in the prediction of PCa detection among patients who underwent initial PBX. The authors reported that PSA-MR was an independent predictor of PCa detection, and was significantly more accurate than PSA. However, the replacement of PSA level with PSA-MR or the addition of PSA-MR in the multivariate model was not associated with a significantly increased accuracy for predicting PCa detection via initial PBx. These results are similar to ours, which showed that PSA-MR was an independent predictor of $\mathrm{PCa}$ detection during repeated PBx. On the other hand, there are several different points between our study and that by Hong et al. (22). The previous study only analyzed initial PBX data, while we analyzed data from a repeated PBx database. Since approximately 30\% of PCa cases are missed during the initial $\mathrm{PBx}$, the previous study was limited in terms of a precise reflection of the clinical utility of PSA-MR. Our analysis of results from a repeated $\mathrm{PBx}$ database could strengthen our understanding of the clinical utility of PSA-MR. Furthermore, since it is more difficult to decide whether to pursue a repeated $\mathrm{PBx}$, compared to an initial $\mathrm{PBx}$, it is more important to identify the parameters associated with the repeated PBx outcome in the clinical setting. Another point is that we analyzed not only the predictive accuracy, but also other predictive parameters such as specificity, sensitivity, PPV, NPV and PAB. Our study showed that the specificity of the model that incorporated PSA-MR was superior to that of the model that did not incorporate PSA-MR. Furthermore, use of the model that incorporated PSA-MR would have significantly avoided unnecessary repeated PBx, even when the models were compared at fixed sensitivity thresholds of 90\% and 95\%. As aforementioned, the avoidance of unnecessary $\mathrm{PBx}$ is as important as $\mathrm{PCa}$ detection, especially when a repeated $\mathrm{PBx}$ is pursued.

Another strength of our study is that it is the first to compare PSA-MR with PSA and its derivatives, considering that PSA-MR is also a PSA derivative. In a logistic regression analysis that included PSA and its derivatives, PSA-MR was an independent predictor of $\mathrm{PCa}$ detection during repeated PBx. From the viewpoint of predictive accuracy, PSA-MR was superior to PSA or most PSA derivatives. Therefore, PSA-MR might be a useful tool for predicting $\mathrm{PCa}$ during repeated $\mathrm{PBx}$.

One fundamental limitation is that PSA-MR is derived from PSA, which is not a cancer-specific marker. Therefore, PSA-MR is not free from the drawbacks associated with PSA, and this might atte- 
nuate the value of PSA-MR. However, the clinical utility of PSA-MR cannot be entirely discounted because there are similar limitations for other PSA derivatives, which are already widely used. PSA-MR is the logical modification of PSA, as it is adjusted by the BMI, plasma volume, and prostate volume. Additionally, our study showed that PSA-MR was an independent predictor of PCa detection, and its use would have avoided 59.6\% of unnecessary repeated PBx. Its predictive accuracy was also superior to that of most other PSA-related parameters, including PSA or PSA-D. Accordingly, PSA-MR is another potentially useful tool for predicting PCa during repeated $\mathrm{PBx}$ that could help physicians to counsel patients regarding further PBx.

Our study had a few limitations. First, not all patients with a negative initial $\mathrm{PBx}$ were included in our study, resulting in a selection bias, although almost all previous studies in this field had the same limitation. However, we analyzed all data at a single institution to reduce the bias. Second, since all of our study subjects were Korean men, the PCa-related clinical characteristics might differ from those of other races (23). Therefore, our results may not be directly applicable to other races. Lastly, new biomarkers such as prostate cancer gene-3 were not analyzed, although the clinical utilities of these new biomarkers have yet to be fully determined (1). However, the most widely used predictors of PCa detection were analyzed.

\section{CONCLUSIONS}

PSA-MR was an independent predictor, and its use would have avoided 59.6\% of unnecessary repeated PBx for PCa detection after a negative initial PBx. PSA-MR was also superior to PSA or most of its derivatives, including PSA-D. Our results support the use of PSA-MR as a helpful tool when counseling patients after a negative initial $\mathrm{PBx}$, and as a means to reduce further unnecessary biopsies.

\section{ABBREVIATIONS}

$\mathrm{PCa}=$ prostate cancer

$\mathrm{PBx}=$ prostate biopsy

PSA = prostate-specific antigen

PSA-MR = psa mass ratio
PSA-V = psa velocity

PSA-D = psa density

PSA-DT = psa doubling time

$\%$ fPSA = percent of free PSA

DRE $=$ digital rectal examination

TRUS $=$ transrectal ultrasonography

HGPIN = high grade prostatic intraepithelial neoplasia

ASAP $=$ atypical small acinar proliferation

$\mathrm{BMI}=$ body mass index

$\mathrm{IR}=$ insulin resistance

$\mathrm{PPV}=$ positive predictive value

$\mathrm{NPV}=$ Negative predictive value

$\mathrm{PAB}=$ Proportion that avoided unnecessary repeated $\mathrm{PBX}$

\section{CONFLICT OF INTEREST}

None declared.

\section{REFERENCES}

1. Heidenreich A, Bellmunt $J$, Bolla $M$, Joniau S, Mason M, Matveev V, et al.: EAU guidelines on prostate cancer. Part 1: screening, diagnosis, and treatment of clinically localised disease. Eur Urol. 2011; 59: 61-71.

2. Zaytoun OM, Jones JS: Prostate cancer detection after a negative prostate biopsy: lessons learnt in the Cleveland Clinic experience. Int J Urol. 2011; 18: 557-68.

3. Benecchi L, Pieri AM, Melissari M, Potenzoni M, Pastizzaro CD: A novel nomogram to predict the probability of prostate cancer on repeat biopsy. J Urol. 2008; 180: 146-9.

4. Scattoni V, Maccagnano C, Zanni G, Angiolilli D, Raber $\mathrm{M}$, Roscigno $\mathrm{M}$, et al.: Is extended and saturation biopsy necessary? Int J Urol. 2010; 17: 432-47.

5. Auprich M, Augustin H, Budäus L, Kluth L, Mannweiler S, Shariat SF, et al.: A comparative performance analysis of total prostate-specific antigen, percentage free prostatespecific antigen, prostate-specific antigen velocity and urinary prostate cancer gene 3 in the first, second and third repeat prostate biopsy. BJU Int. 2012; 109: 1627-35.

6. Catalona WJ, Southwick PC, Slawin KM, Partin AW, Brawer MK, Flanigan RC, et al.: Comparison of percent free PSA, PSA density, and age-specific PSA cutoffs for prostate cancer detection and staging. Urology. 2000; 56: 255-60.

7. Choi HC, Park JH, Cho BL, Son KY, Kwon HT: Prostate specific antigen mass ratio potential as a prostate cancer screening tool. J Urol. 2010; 184: 488-93. 
8. Klein LT, Lowe FC: The effects of prostatic manipulation on prostate-specific antigen levels. Urol Clin North Am. 1997; 24: 293-7.

9. Marks LS, Andriole GL, Fitzpatrick JM, Schulman CC, Roehrborn CG: The interpretation of serum prostate specific antigen in men receiving 5alpha-reductase inhibitors: a review and clinical recommendations. J Urol. 2006; 176: 868-74.

10. Fowler JE Jr, Bigler SA, Kilambi NK, Land SA: Relationships between prostate-specific antigen and prostate volume in black and white men with benign prostate biopsies. Urology. 1999; 53: 1175-8.

11. Nogueira L, Corradi R, Eastham JA: Prostatic specific antigen for prostate cancer detection. Int Braz J Urol. 2009; 35: 5219; discussion 530-2.

12. Barqawi AB, Golden BK, O'Donnell C, Brawer MK, Crawford ED: Observed effect of age and body mass index on total and complexed PSA: analysis from a national screening program. Urology. 2005; 65: 708-12.

13. Connolly D, Black A, Murray LJ, Napolitano G, Gavin A, Keane PF: Methods of calculating prostate-specific antigen velocity. Eur Urol. 2007; 52: 1044-50.

14. Ramírez ML, Nelson EC, Devere White RW, Lara PN Jr, Evans CP: Current applications for prostate-specific antigen doubling time. Eur Urol. 2008; 54: 291-300.

15. Du Bois D, Du Bois EF: A formula to estimate the approximate surface area if height and weight be known. 1916. Nutrition. 1989; 5: 303-11; discussion 312-3.

16. Boer $P$ : Estimated lean body mass as an index for normalization of body fluid volumes in humans. Am J Physiol. 1984; 247: F632-6.

17. Bañez LL, Hamilton RJ, Partin AW, Vollmer RT, Sun L, Rodriguez $\mathrm{C}$, et al.: Obesity-related plasma hemodilution and PSA concentration among men with prostate cancer. JAMA. 2007; 298: 2275-80.
18. Stamey TA, Yang N, Hay AR, McNeal JE, Freiha FS, Redwine E: Prostate-specific antigen as a serum marker for adenocarcinoma of the prostate. N Engl J Med. 1987; 317: 909-16.

19. Thompson IM, Tangen CM, Ankerst DP, Chi C, Lucia MS, Goodman P, et al.: The performance of prostate specific antigen for predicting prostate cancer is maintained after a prior negative prostatebiopsy. J Urol. 2008; 180: 544-7.

20. Catalona WJ, Partin AW, Slawin KM, Brawer MK, Flanigan $\mathrm{RC}$, Patel A, et al.: Use of the percentage of free prostatespecific antigen to enhance differentiation of prostate cancer from benign prostatic disease: a prospective multicenter clinical trial. JAMA. 1998; 279: 1542-7.

21. Keetch DW, McMurtry JM, Smith DS, Andriole GL, Catalona WJ: Prostate specific antigen density versus prostate specific antigen slope as predictors of prostate cancer in men with initiallynegative prostatic biopsies. J Urol. 1996; 156: 428-31.

22. Hong SK, Oh JJ, Byun SS, Hwang SI, Choo MS, Lee SE: Value of prostate-specific antigen (PSA) mass ratio in the detection of prostate cancer in men with PSA levels of $\leq 10$ ng/mL. BJU Int. 2012; 110: E81-5.

23. Lee DH, Lee SH, Rha KH, Choi IY, Lee JY, Kim SW, et al.: The Establishment of K-CaP (the Multicenter Korean Prostate Cancer Database). Korean J Urol. 2013; 54: 229-33.

Correspondence address: Seok-Soo Byun, MD, PhD Department of Urology, Seoul National University Bundang Hospital 166, Gumi-ro, Bundang-gu Seongnam, Kyunggi-do, 463-707, Korea Fax: + 8231 787-4057 E-mail: ssbyun@snubh.org 